\title{
Velocity-independent and low- complexity method for 1D DOA estimation using an arbitrary cross-linear array
}

\author{
Gengxin Ning ${ }^{1 *}$, Guangyu Jing ${ }^{1}$, Xiaopeng $\mathrm{Li}^{1}$ and Xuejin Zhao ${ }^{2}$
}

\begin{abstract}
This paper focuses on a low-complexity one-dimensional (1D) direction-of-arrival (DOA) algorithm with an arbitrary cross-linear array. This algorithm is highly accurate without the performance error usually caused by the uncertainty factor of the wave velocity in the underwater environment. The geometric relationship between two crossed linear arrays is employed to derive the expression of DOA estimation with the finding that this algorithm is capable of excluding the wave velocity variable in the DOA estimation expression. A method without parameter pairing is also proposed to reduce the complexity of this algorithm. Additionally, the influence of wave velocity is analyzed in terms of $R M S E_{C}$ and the Cramer-Rao bound (CRB) for 1D DOA with the arbitrary cross-linear array is established. The simulation results demonstrate that the proposed algorithm can achieve better performance than the traditional algorithm under the condition of an inaccurate estimate of wave velocity. Compared with the velocity-independent DOA algorithm, it exhibits the feature of low complexity.
\end{abstract}

Keywords: 1D-DOA, Velocity independent, Arbitrary cross-linear array, Cramer-Rao bound

\section{Introduction}

The estimation of the underwater DOA is widely used in many fields, such as underwater target positioning, offshore operations, and military reconnaissance [1]. Compared with an electromagnetic wave, a sound wave undergoes less loss in underwater propagation. Therefore, underwater DOA estimation usually employs sound waves as communication signals and takes wave velocity as a constant. However, in a real underwater environment, the numerical value of wave velocity constantly changes for it is affected by temperature, salinity, and other factors [2]. The actual amount of wave velocity in seawater approximately ranges from 1450 to $1550 \mathrm{~m} / \mathrm{s}$. The assumption that the wave velocity is a constant will generate serious errors. Therefore, it is critical to handle the influence factors of the wave velocity in underwater DOA estimation.

*Correspondence: ninggx@scut.edu.cn

${ }^{1}$ South China University of Technology, Guangzhou, China

Full list of author information is available at the end of the article
The ESPRIT algorithm [3] has been extensively used since it was proposed because, among other characteristics, it features no spectral peak search and low computational requirements. Several enhanced algorithms are based on the ESPRIT algorithm. Qian has proposed the MR-ESPRIT algorithm [4], which improved the array division of the classical ESPRIT algorithm. This classical algorithm sets down two sub-arrays with an offset of 1 on a single linear array. It then uses the rotation operator between the two sub-arrays to obtain the result. By contrast, the MR-ESPRIT algorithm divides sub-arrays with an offset greater than 1 . It utilizes the received signals of the two sub-arrays to restore the direction matrix of the array. Finally, the estimation result is obtained by solving the direction matrix. The MR-ESPRIT algorithm is superior at suppressing the impact of noise. An automatic pairing joint direction-of-arrival and frequency estimation, abbreviated as AF-ESPRIT, is presented in [5]. By using the multiple-delay output of a uniform linear antenna array (ULA), this algorithm can estimate joint 
angles and frequencies. In [6], a frequency-angle joint estimation algorithm is proposed. It is based on singular value decomposition (SVD) and trilinear decomposition. This algorithm eliminates the influence of the number of snapshots on the computational complexity through SVD and reduces the estimation complexity without losing estimation performance. Pinto [7] and others proposed an estimation algorithm abbreviated as MS-KAI-ESPRIT that performs better at a low signal-to-noise ratio (SNR) and small amounts of snapshots. First, this algorithm sets a convergence factor and roughly estimates the DOA of the source signal through the classical ESPRIT algorithm. Next, it uses this result to correct the data covariance matrix and utilizes the modified covariance matrix to estimate the new DOA angle; cyclic iteration is then applied until the convergence condition is satisfied. Onedimensional DOA estimation with different structured arrays, such as L-shaped arrays [8-10] and a uniform rectangular array, has captured a remarkable amount of attention. The MUSIC algorithm has also aroused notable research interest [11-14] given that it has a high resolution, estimation accuracy, and stability under certain conditions. In [15], a velocity-independent MUSIC algorithm (VI-MUSIC) is proposed. It is based on L-shaped arrays. The VI-MUSIC algorithm uses an L-shaped array and the so-called Same Peak method to eliminate the effect of using imprecise wave velocity. This algorithm can achieve high accuracy even though imprecise wave velocity is used for DOA estimation.

However, the real-time propagation velocity of the sound wave is unknown because of the instability and complexity of the underwater environment. The ESPRIT algorithms mentioned above calculate the wave velocity as a constant. Adopting imprecise velocity leads to a significant error. Despite being a velocity-independent algorithm, the previously mentioned VI-MUSIC algorithm needs to search for real-time wave velocity in the range of $1450-1550 \mathrm{~m} / \mathrm{s}$, which implies high and often unacceptable computational complexity.

This paper proposes a $1 \mathrm{D}$ velocity independent and low complexity ESPRIT algorithm (VI-LC-ESPRIT) based on an arbitrary cross-linear array to solve the above problem. The proposed algorithm uses the geometric relationship between the arbitrary cross-linear array to eliminate the effect of variable wave velocity. Besides, it exhibits low computational complexity without parameter matching.

\section{Signal model and wave velocity influence analysis}

2.1 Arbitrary cross-linear array structure and data model As illustrated in Fig.1, $K$ far-field narrowband plane wave signals $s_{i}(t), i=1, \ldots, K$, impinge on the arbitrary crosslinear array. Arbitrary cross-linear array is structured by two uniform arrays in the $x-y$ plane with an cross angle

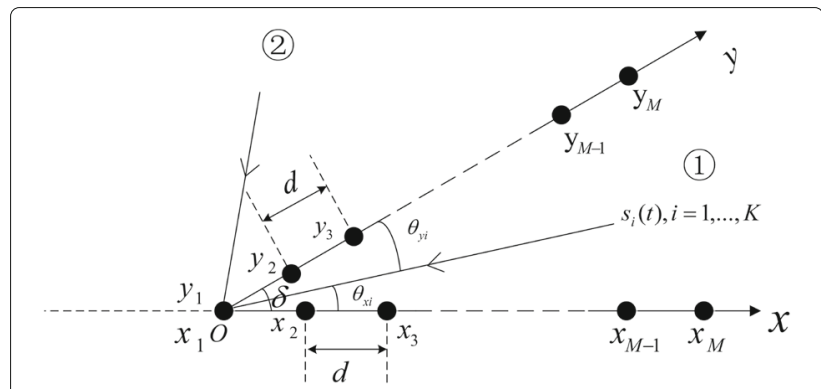

Fig. 1 Arbitrary cross-linear array for 1D DOA estimation

of $\delta$, where $\delta \in\left(0, \frac{\pi}{2}\right)$. Each array consists of $M$ identical omni-directional sensors separated by inter-element spacing $d$, namely, $d=\lambda / 2$, where $\lambda$ approximates to be the wavelength of the incident waves. The total number of sensors is $N-1$, where $N=2 M \cdot \mathbf{r}_{n}=\left[x_{n}, y_{n}\right]^{\mathrm{T}}, n=$ $1, \ldots, N$ is the position vector of sensor $n$. The position of sensor $m$ on the $x$-axis is denoted as $r_{m}, m=1, \ldots, M$, and the position of sensor $m$ on the $y$-axis is denoted as $r_{m+M}$. The center frequency of the $i$ th signal is $f_{i}$, and considering the underwater homogeneous isotropic fluid medium environment, their wave velocity is defined as $c$, where $c \in[1450,1550] \mathrm{m} / \mathrm{s}$. Let us note $\theta_{x i}$ the DOA of the $i$ th signal on the $x$-axis, where $\theta_{x i} \in(0, \pi)$, and $\theta_{y i}$ the DOA of the $i$ th signal on the $y$-axis, where $\theta_{y i} \in(0, \pi)$.

Using the array element at $O$ as the reference element. As shown in Fig. 1, the incoming area of the source signals is divided into two areas. In area (1), we have $\theta_{y i}=\delta-\theta_{x i}$, and in area (2), we have $\theta_{y i}=\theta_{x i}-\delta$. That is to say, in 1D-DOA estimation, the angles between the narrowband signal and arbitrary cross-linear array satisfy

$$
\cos \theta_{y i}=\cos \left(\theta_{x i}-\delta\right),
$$

where $\theta_{x i}=\theta_{i}$, and $\theta_{i}$ is the expected wave direction angle of the $i$ th source signal.

The received signals of the array on the $x$-axis and the $y$-axis are written as

$$
\begin{aligned}
& \mathbf{X}(t)=\mathbf{A}_{x} \mathbf{S}(t)+\mathbf{N}_{x}(t), \\
& \mathbf{Y}(t)=\mathbf{A}_{y} \mathbf{S}(t)+\mathbf{N}_{y}(t) .
\end{aligned}
$$

where $\mathbf{S}(t)=\left[s_{1}(t), s_{2}(t), \ldots, s_{k}(t)\right]^{\mathrm{T}}$ is the $K \times 1$ incoming source signals vector, $\mathbf{N}_{x}(t)$ and $\mathbf{N}_{y}(t)$ are the Gaussian white noise vectors along the $x$-axis and the $y$-axis, respectively. The $M \times K$ array manifold matrices $\mathbf{A}_{x}$ and $\mathbf{A}_{y}$ can be represented as

$$
\begin{aligned}
& \mathbf{A}_{x}=\left[\mathbf{a}\left(\theta_{x 1}\right), \mathbf{a}\left(\theta_{x 2}\right), \ldots, \mathbf{a}\left(\theta_{x K}\right)\right], \\
& \mathbf{A}_{y}=\left[\mathbf{a}\left(\theta_{y 1}\right), \mathbf{a}\left(\theta_{y 2}\right), \ldots, \mathbf{a}\left(\theta_{y K}\right)\right] .
\end{aligned}
$$

We define $\tau_{n}\left(\theta_{x i}\right)=\mathbf{d}^{\mathrm{T}}\left(\theta_{x i}\right) \cdot \mathbf{r}_{n} / c$ as the propagation delay of the $i$ th signal received sensor $n$, where $\mathbf{d}\left(\theta_{x i}\right)=$ $\left[\cos \theta_{x i}, \sin \theta_{x i}\right]^{\mathrm{T}}$ is the unit vector pointing towards the $i$ th signal, and $\mathbf{r}_{n}$ is the position vector of sensor $n$. 


$$
\begin{aligned}
& \mathbf{a}\left(\theta_{x i}\right)=\left[a_{1}\left(\theta_{x i}\right), a_{2}\left(\theta_{x i}\right), \ldots, a_{M}\left(\theta_{x i}\right)\right]^{\mathrm{T}}, \\
& \mathbf{a}\left(\theta_{y i}\right)=\left[a_{1}\left(\theta_{y i}\right), a_{2}\left(\theta_{y i}\right), \ldots, a_{M}\left(\theta_{y i}\right)\right]^{\mathrm{T}},
\end{aligned}
$$

are denoted as $M \times 1$ array manifold vectors, which have the form of $a_{m}\left(\theta_{x i}\right)=e^{-j 2 \pi f_{i} \tau_{m}\left(\theta_{x i}\right)}$ on the $x$-axis and $a_{m}\left(\theta_{y i}\right)=e^{-j 2 \pi f_{i} \tau_{m+M}\left(\theta_{y i}\right)}$ on the $y$-axis, respectively. We suppose that the source signals are non-Gaussian and uncorrelated to each other. And the Gaussian noises with zero-mean and variance $\sigma^{2}$ are assumed to be statistically independent to the signals.

\subsection{Analysis of the wave velocity influence}

After performing 1D Root-Music algorithm or TLSESPRIT algorithm, the roots $u_{i}$ on the $x$-axis and $v_{i}$, $i=1, \ldots, K$ on the $y$-axis are obtained. The relationship between $u_{i}, v_{i}, \theta_{x i}$ and the real-time underwater wave velocity $c$ are expressed as follows.

$$
\begin{aligned}
& \arg \left(u_{i}\right)=-2 \pi d \cos \theta_{x i} f_{i} / c, \\
& \arg \left(v_{i}\right)=-2 \pi d \cos \theta_{y i} f_{i} / c .
\end{aligned}
$$

According to Eq. (5), the DOA can be rewritten as

$$
\begin{aligned}
& \hat{\theta}_{x i}=\arccos \left(-\arg \left(u_{i}\right) c / 2 \pi d f_{i}\right), \\
& \hat{\theta}_{y i}=\arccos \left(-\arg \left(v_{i}\right) c / 2 \pi d f_{i}\right) .
\end{aligned}
$$

Traditional algorithms such as TLS-ESPRIT, RootMUSIC, and MS-KAI-ESPRIT perform well on the premise conditions: (1) DOA and frequency parameters are paired; (2) the real-time wave velocity is $1500 \mathrm{~m} / \mathrm{s}$. The AF-ESPRIT algorithm can obtain automatic pairing DOA and frequency parameters by using the multipledelay output of a uniform linear antenna array, and it performs well when real-time wave velocity is $1500 \mathrm{~m} / \mathrm{s}$. In summary, the above algorithms must set a constant value of $c_{0}$ as the real-time wave velocity, where $c_{0}=1500 \mathrm{~m} / \mathrm{s}$.

However, the real-time wave velocity $\mathrm{c}$ is a variable in the range of $1450-1550 \mathrm{~m} / \mathrm{s}$. Let $\Delta c=c-c_{0}$. According to Eq. (6), the greater $|\Delta c|$ is, the greater deviation is in DOA estimation comparing to the estimation when $|\Delta c|=0$. In order to evaluate the impact of using inaccurate wave velocity c0 instead of real-time wave velocity c, we define $R M S E_{c}$ using different real-time wave velocity values.

$$
\begin{aligned}
\operatorname{RMSE}_{c} & =\frac{1}{K} \sum_{i=1}^{K} \sqrt{\left(\theta_{x i}-\hat{\theta}_{x i}\right)^{2}} \\
& =\frac{1}{K} \sum_{i=1}^{K}\left|\theta_{x i}-\arccos \frac{c_{0} \cos \theta_{x i}}{c}\right|
\end{aligned}
$$

\section{Method of velocity-independent and low-complexity DOA estimation}

\subsection{The VI-LC-ESPRIT algorithm}

Firstly, a cross-correlation matrix $\mathrm{R}_{x y}$ is obtained by Eq. (8).

$$
\mathbf{R}_{x y}=\mathrm{E}\left[\mathbf{X}(t) \mathbf{Y}^{\mathrm{H}}(t)\right]=\mathbf{A}_{x} \mathbf{R}_{s} \mathbf{A}_{y},
$$

where $\mathbf{R}_{s}=\mathrm{E}\left\{\mathbf{S}(t) \mathbf{S}^{\mathrm{H}}(t)\right\}$. It can be noted that the additive noise is removed by the cross-correlation operation. Let $\mathbf{R}_{x y 1}$ and $\mathbf{R}_{x y 2}$ be the first and last $M-1$ cols of $\mathbf{R}_{x y}$, respectively, so

$$
\begin{aligned}
& \mathbf{R}_{x y 1}=\mathbf{A}_{x} \mathbf{R}_{s} \mathbf{A}_{y 1}^{\mathrm{H}}, \\
& \mathbf{R}_{x y 2}=\mathbf{A}_{x} \mathbf{R}_{s} \mathbf{A}_{y 2}^{\mathrm{H}},
\end{aligned}
$$

In the equation above, $\mathbf{A}_{y 2}=\mathbf{A}_{y 1} \Omega^{\mathrm{H}}, \mathbf{A}_{y 1}$ and $\mathbf{A}_{y 2}$ are defined as the first and last $M-1$ rows of $\mathbf{A}_{y}$, and $\Omega=\operatorname{diag}\left\{e^{j 2 \pi d f_{1} \cos \theta_{y 1} / c}, \ldots, e^{j 2 \pi d f_{K} \cos \theta_{y K} / c}\right\}$. Since the $K$ source signals are uncorrelated, it is easy to know that $\mathbf{A}_{y 1}$, $\mathbf{A}_{y 2}$ and $\mathbf{R}_{s}$ are full rank matrices.

By combining Eq. (9), a new $2 M \times(M-1)$ matrix $\mathbf{R}$ is defined by using Eq. (10).

$$
\mathbf{R}=\left[\begin{array}{l}
\mathbf{R}_{x y, 1} \\
\mathbf{R}_{x y, 2}
\end{array}\right]=\left[\begin{array}{c}
\mathbf{A}_{x} \\
\mathbf{A}_{x} \Omega
\end{array}\right] \mathbf{R}_{s} \mathbf{A}_{y 1}^{\mathrm{H}}=\mathbf{B} \mathbf{R}_{s} \mathbf{A}_{y 1}^{\mathrm{H}},
$$

where a new direction matrix $\mathbf{B}$ can be expressed as

$$
\mathbf{B}=\left[\begin{array}{c}
\mathbf{A}_{x} \\
\mathbf{A}_{x} \Omega
\end{array}\right] \text {. }
$$

We can obtain the singular value from the decomposition of matrix $\mathbf{R}$ using

$$
\mathbf{R}=\mathbf{U} \Sigma \mathbf{V}^{\mathrm{H}}=\left[\mathbf{U}_{s}, \mathbf{U}_{n}\right]\left[\begin{array}{cc}
\Sigma_{s} & 0 \\
0 & \Sigma_{n}
\end{array}\right]\left[\begin{array}{l}
\mathbf{V}_{s}^{\mathrm{H}} \\
\mathbf{V}_{n}^{\mathrm{H}}
\end{array}\right],
$$

where $\Sigma_{s}$ is a $K \times K$ matrix, $\mathbf{U}_{s}$ is the signal subspace, and $\mathbf{U}_{n}$ is the noise subspace. By combining Eq. (8), it can be noted that $\Sigma_{n}=\mathbf{0}$. In addition, $\mathbf{U}=\left[\mathbf{U}_{s}, \mathbf{U}_{n}\right]$ is a unitary matrix, so it can be obtained by

$$
\mathbf{U}_{s}^{\mathrm{H}} \mathbf{U}_{n}=\mathbf{0} \text {. }
$$

According to the properties of SVD, we have

$$
\left(\mathbf{R} \mathbf{V}_{n}\right)^{\mathrm{H}} \mathbf{U}_{n}=\left(\mathbf{U}_{n} \Sigma_{n}\right)^{\mathrm{H}} \mathbf{U}_{n},
$$

Considering $\mathbf{V}_{n}$ is a full-rank matrix and $\Sigma_{n}=\mathbf{0}$, and combining Eqs. (10), (14) can be rewritten as

$$
\mathbf{R}^{\mathrm{H}} \mathbf{U}_{n}=\mathbf{A}_{y 1} \mathbf{R}_{s}^{\mathrm{H}} \mathbf{B}^{\mathrm{H}} \mathbf{U}_{n}=\mathbf{0} .
$$

It is easy to know that $\mathbf{A}_{y 1} \mathbf{R}_{s}^{\mathrm{H}}$ is a full-rank matrix. So, $\mathbf{B}$ and $\mathbf{U}_{n}$ have the following relationship.

$$
\mathbf{B}^{\mathrm{H}} \mathbf{U}_{n}=\mathbf{0} \text {. }
$$

By combining Eqs. (13) and (16), a non-singular matrix $\mathbf{T}$ is defined by Eq. (17).

$$
\mathbf{U}_{s}=\mathbf{B} \mathbf{T} \text {. }
$$

Then, sub-matrices $\mathbf{B}_{1}$ and $\mathbf{B}_{\mathbf{2}}$ are defined using

$$
\begin{aligned}
& \mathbf{B}_{1}=\left[\begin{array}{c}
\mathbf{B}(1: M-1,:) \\
\mathbf{B}(M+1: 2 M-1,:)
\end{array}\right], \\
& \mathbf{B}_{2}=\left[\begin{array}{c}
\mathbf{B}(2: M,:) \\
\mathbf{B}(M+2: 2 M,:)
\end{array}\right] .
\end{aligned}
$$


There is a matrix $\Psi$ that satisfies

$$
\mathbf{B}_{2}=\mathbf{B}_{1} \Psi,
$$

where $\Psi=\operatorname{diag}\left\{\Psi_{1}, \cdots, \Psi_{K}\right\} . \Psi$ is called the rotation matrix, whereas $\Psi_{i}$ is called a phase rotation operator, and $\Psi_{i}=e^{-j 2 \pi d f_{i} \cos \theta_{x i} / c} . \theta_{x i}$ can be obtained if $\Psi_{i}$ is determined. The matrix $\mathbf{U}_{s}$ is divided into matrix $\mathbf{U}_{1}$ and $\mathbf{U}_{2}$ in the same way as

$$
\begin{aligned}
\mathbf{U}_{1} & =\left[\begin{array}{c}
\mathbf{U}_{s}(1: M-1,:) \\
\mathbf{U}_{s}(M+1: 2 M-1,:)
\end{array}\right], \\
\mathbf{U}_{2} & =\left[\begin{array}{c}
\mathbf{U}_{s}(2: M,:) \\
\mathbf{U}_{s}(M+2: 2 M,:)
\end{array}\right]
\end{aligned}
$$

According to Eq. (17), we obtain

$$
\begin{aligned}
& \mathbf{U}_{1}=\mathbf{B}_{1} \mathbf{T}, \\
& \mathbf{U}_{2}=\mathbf{B}_{2} \mathbf{T},
\end{aligned}
$$

By combining Eqs. (19) and (21), there is

$$
\mathbf{U}_{2}=\mathbf{B}_{1} \Psi \mathbf{T}=\mathbf{B}_{1} \mathbf{T} \mathbf{T}^{-1} \Psi \mathbf{T}=\mathbf{U}_{1} \mathbf{T}^{-1} \Psi \mathbf{T} .
$$

$\mathbf{U}_{1}^{+}$is defined as the Moore-Penrose generalized inverse of $\mathbf{U}_{1}$, and Eq. (22) can be rewritten adopting

$$
\mathbf{U}_{1}^{+} \mathbf{U}_{2}=\mathbf{T}^{-1} \Psi \mathbf{T} \text {. }
$$

In Eq. (23), by performing eigenvalue decomposition (EVD) of $\mathbf{U}_{1}^{+} \mathbf{U}_{2}$, eigenvalues $\lambda_{1}, \lambda_{2}, \cdots, \lambda_{K}$ and corresponding eigenvectors $\mathbf{T}^{-1}$ are obtained. Besides, $\lambda_{1}, \lambda_{2}, \cdots, \lambda_{K}$ correspond to the diagonal elements of $\Psi$. Then, according to the expression of $\Psi$, the azimuth angles can be expressed as

$$
\cos \theta_{x i}=\frac{\arg \lambda_{i}}{-2 \pi d f_{i} / c}, i=1,2, \cdots, K .
$$

Here, we obtain the estimated value of the direction matrix $\mathbf{B}$ adopting

$$
\widehat{\mathbf{B}}=\mathbf{U}_{s} \mathbf{T}^{-1} .
$$

In addition, combining Eq. (11) and the expression of $\Omega, \mathbf{B}(1, i) \mathbf{B}^{*}(M+1, i)=e^{-j 2 \pi d f_{i} \cos \theta_{y i} / c}$ can be obtained, where $\mathbf{B}(k, i)$ is the element of the $k$ th row and the $i$ th col of $\mathbf{B}$. That is to say

$$
\cos \theta_{y i}=\frac{\arg \left(\widehat{\mathbf{B}}(1, i) \widehat{\mathbf{B}}^{*}(M+1, i)\right)}{-2 \pi d f_{i} / c}, i=1,2, \cdots, K
$$

where $\widehat{\mathbf{B}}(k, i)$ is the element of the $k$ th row and the $i$ th col of $\widehat{\mathbf{B}}$. The effect of inaccurate wave velocity can be reduced by dividing Eqs. (24) and (26).

$$
\frac{\cos \theta_{y i}}{\cos \theta_{x i}}=\frac{\arg \left(\widehat{\mathbf{B}}(1, i) \widehat{\mathbf{B}}^{*}(M+1, i)\right)}{\arg \left(\lambda_{i}\right)}, i=1,2, \cdots, K .
$$

Finally, combined with Eq. (1), the azimuth angles can be obtained as follows.

$$
\theta_{i}=\tan ^{-1}\left(\frac{\arg \left(\widehat{\mathbf{B}}(1, i) \widehat{\mathbf{B}}^{*}(M+1, i)\right)}{\arg \left(\lambda_{i}\right) \sin \delta}-\cot \delta\right),
$$

when $\arg \left(\lambda_{i}\right) \neq 0$, and $\theta_{i}=0$ when $\arg \left(\lambda_{i}\right)=0$.

Remark: According to the definition of $\Omega$ and the construction method of the matrix $\mathbf{B}$ in Eq. (11), it can be found that the column vectors of $\mathbf{B}$ only contain paired $\theta_{x i}$ and $\theta_{y i}$, where $\cos \theta_{y i}=\cos \left(\theta_{x i}-\delta\right)$. From Eqs. (23) and (25), $\mathbf{U}_{1}^{+} \mathbf{U}_{2}=\mathbf{T}^{-1} \Psi \mathbf{T}$, and the estimated value of $\mathbf{B}$ satisfies $\widehat{\mathbf{B}}=\mathbf{U}_{s} \mathbf{T}^{-1}$, so the columns of $\widehat{\mathbf{B}}$ and $\Psi$ have related permutations. Therefore, $\cos \theta_{x i}$ and $\cos \theta_{y i}$ are matched in Eq. (27) according to Eqs. (23) to (26).

The summary of the proposed VI-LC-ESPRIT algorithm is shown as follows:

Step 1: Compute $\mathbf{R}_{x y}$ and construct $\mathbf{R}$ from Eqs. (8) and (10);

Step 2: Construct $\mathbf{U}_{s}, \mathbf{U}_{1}$, and $\mathbf{U}_{2}$ from Eqs. (12) and (20); Step 3: Estimate $\lambda_{i}$ and $\widehat{\mathbf{B}}$ from Eqs. (23) and (25);

Step 4: Obtain velocity independent azimuth angle from Eq. (28).

\subsection{Cramer-Rao bound (CRB) analysis}

When the data vector is assumed to be Gaussian distributed, a particularly convenient CRB formula is derived in reference [16]. In case of L-shaped array configuration, the CRB formula of 2D DOAs is given in reference [17], where the wave velocity is taken as a constant. The CRB formula of the 1D DOAs using an arbitrary cross-line array is considered here, taking into account the variable wave velocity. Construct a new received data matrix from the arbitrary cross-linear array as follows.

$$
\mathbf{Z}(t)=\left[\begin{array}{l}
\mathbf{X}(t) \\
\mathbf{Y}(t)
\end{array}\right]=\left[\begin{array}{l}
\mathbf{A}_{x}(\theta) \\
\mathbf{A}_{y}(\theta)
\end{array}\right] \mathbf{S}(t)+\left[\begin{array}{l}
\mathbf{N}_{x}(t) \\
\mathbf{N}_{y}(t)
\end{array}\right]
$$

Equation (29) can be rewritten adopting

$$
\mathbf{Z}(t)=\mathbf{A S}(t)+\mathbf{N} .
$$

The Fisher information matrix $\mathbf{F}$ is with respect to $\theta=$ $\left[\theta_{1}, \theta_{2}, \cdots, \theta_{K}\right]$. The $i$,jth element of $\mathbf{F}$ is

$$
\begin{aligned}
\mathbf{F}_{i j} & =2 \operatorname{Re}\left\{\operatorname{trace}\left[\left(\frac{\partial \mathbf{A}}{\partial \theta_{i}} \mathbf{S}\right)^{\mathrm{H}} \frac{1}{\sigma^{2}}\left(\frac{\partial \mathbf{A}}{\partial \theta_{j}} \mathbf{S}\right)\right]\right\} \\
& =\frac{2}{\sigma^{2}} \operatorname{Re}\left\{\operatorname{trace}\left[\left(\frac{\partial \mathbf{A}}{\partial \theta_{i}} \mathbf{e}_{i} \mathbf{e}_{i}^{\mathrm{T}} \mathbf{S}\right)^{\mathrm{H}}\left(\frac{\partial \mathbf{A}}{\partial \theta_{j}} \mathbf{e}_{j} \mathbf{e}_{j}^{\mathrm{T}} \mathbf{S}\right)\right]\right\} \\
& =\frac{2}{\sigma^{2}} \operatorname{Re}\left\{\left(\mathbf{e}_{i}^{\mathrm{T}} \frac{\partial \mathbf{A}^{\mathrm{H}}}{\partial \theta} \frac{\partial \mathbf{A}}{\partial \theta} \mathbf{e}_{j}\right)\left(\mathbf{e}_{j}^{\mathrm{T}} \mathbf{S S}^{\mathrm{H}} \mathbf{e}_{i}\right)\right\} \\
& =\frac{2}{\sigma^{2}} \operatorname{Re}\left\{\left(\frac{\partial \mathbf{A}^{\mathrm{H}}}{\partial \theta} \frac{\partial \mathbf{A}}{\partial \theta}\right)_{i j}\left(\mathbf{S S}^{\mathrm{H}}\right)_{i j}\right\}
\end{aligned}
$$


The concrete form of $\mathbf{F}_{i i}$ is

$\mathbf{F}_{i i}=\frac{8 \pi^{2} f_{i}^{2} L}{\sigma^{2} c^{2}} \sum_{i=1}^{K} \sum_{n=1}^{N}\left\{\mathbf{A}_{n i}^{*} \mathbf{A}_{n i}\left(y_{n} \cos \theta_{i}-x_{n} \sin \theta_{i}\right)^{2}\left(\mathbf{R}_{s}\right)_{i i}\right\}$

where $\mathbf{A}_{n i}$ is the $n$, $i$ th element of $\mathbf{A},\left[x_{n}, y_{n}\right]$ is the position of the $n$th sensor $r_{n}, \mathbf{R}_{s}=\mathbf{E}\left[\mathbf{S S}^{\mathrm{H}}\right]$ and $\left(\mathbf{R}_{s}\right)_{i i}$ is the $i, i$ th element of $\mathbf{R}_{s}$.

Then, the CRB matrix can be expressed using

$$
\mathbf{C}=\mathbf{F}^{-1} \text {. }
$$

Let $\mathbf{C}_{i j}$ be the $i$,jth element of $\mathbf{C}$ and we can obtain the $\mathrm{CRB}$ of the $i$ th azimuth angle as Eq. (34).

$$
\mathrm{CRB}_{\theta_{i}}=\sqrt{\mathbf{C}_{i, i}} .
$$

Thus, the total CRB of 1D DOAs can be expressed by Eq. (35).

$$
\mathrm{CRB}=\frac{1}{K} \sum_{i=1}^{K} \mathrm{CRB}_{\theta_{i}} .
$$

According to Eq. (32), it can be found that $\mathbf{F}_{i i}$ increases as snapshots $L$ increases and decreases as wave velocity $c$ increases or noise power $\sigma^{2}$ increases. When $\delta=90^{\circ}$, $\left(y_{n} \cos \theta_{i}-x_{n} \sin \theta_{i}\right)^{2}$ has a maximum value, which means that $\mathbf{F}_{i i}$ has a maximum value. By combining Eqs. (33) and (35), it can be concluded that the CRB of 1D DOAs increases as $c$ increases, decreases as L or SNR increases, and has a minimum value when $\delta=90^{\circ}$.

\subsection{Complexity analysis}

In this section, we mainly compare the performance of the following algorithms: TLS-ESPRIT, Root-MUSIC, AFESPRIT in [5], MS-KAI-ESPRIT in [7], VI-MUSIC in [15], and the proposed VI-LC-ESPRIT algorithm. $K$ is the number of source signals, $N$ is the number of total sensors, and $L$ is the sample snapshots.

As for the complexity, we analyze it based on matrix complex multiplication, which mainly involves in autocorrelation or cross-correlation matrix construction, EVD or SVD operation, and pseudo-inverse operation. Due to $L>>N>K$, we mainly study the relationship between the complexity of the five algorithms and $L, N$. The complexity of the proposed VI-LC-ESPRIT algorithm is about $O\left(M^{2} L+20 M^{3}+4 M^{2} K+2 M K^{2}+K^{3}\right)$, while that of the classical TLS-ESPRIT algorithm is about $O\left(N^{2} L+4 N^{3}\right)$, where $N=2 M$. The running time of the six algorithms is employed to check the performance of complexity. The results of the running times are shown in the following figures.

Figures 2 and 3 show that the complexity of the six algorithms increases gradually with the increase of sensors,

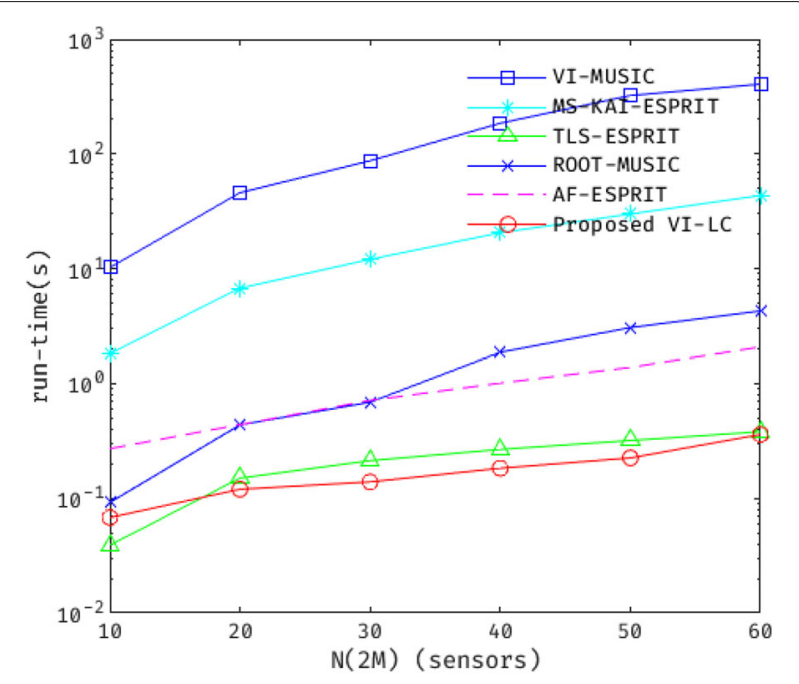

Fig. 2 Complexity comparison versus sensors

but increases slowly with the increase of snapshots. Compared with other algorithms, the VI-MUSIC algorithm and the MS-KAI-ESPRIT algorithm have higher computational complexity. The complexity of the TLS-ESPRIT algorithm is low. In general, the proposed VI-LC-ESPRIT algorithm has lower complexity than others and has an advantage when the wave velocity is unknown. Because the VI-MUSIC algorithm needs to search the real-time wave velocity, its computational complexity is even hundreds of times that of the proposed VI-LC-ESPRIT algorithm. Considering that a large amount of calculation limits the practical value of the VI-MUSIC algorithm, its estimation performance is not compared in Section 4.

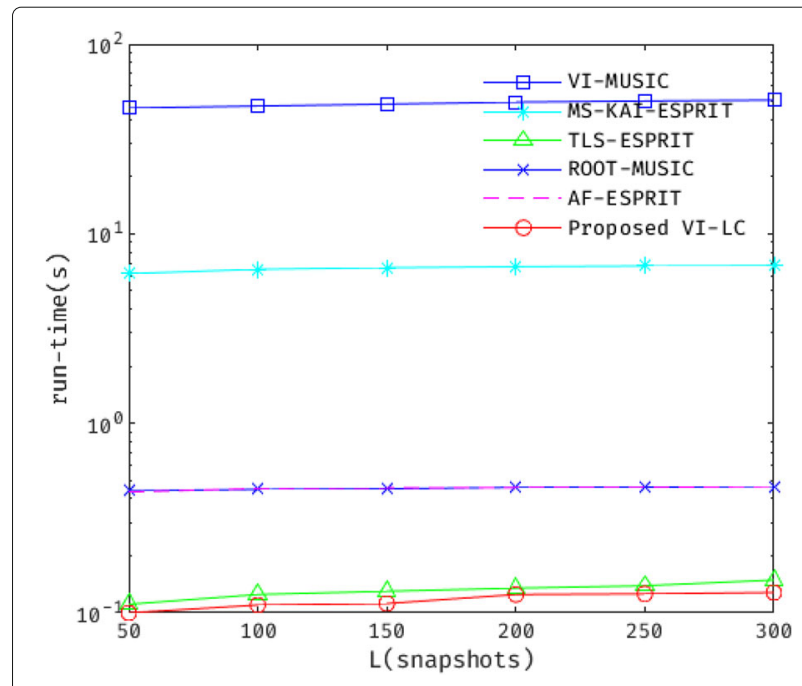

Fig. 3 Complexity comparison versus snapshots 


\section{Simulation results and discussion}

In all simulation experiments, the sampling frequency is $40 \mathrm{kHz}$, the number of snapshots $L$ is 200 , the element spacing $d$ is $0.05 \mathrm{~m}$, and 1000 Monte Carlo trials are conducted. Note that $\Delta c=c-c_{0}$, where $c_{0}=1500 \mathrm{~m} / \mathrm{s}$. The array structure used in the VI-LC-ESPRIT algorithm is a $M \times 2$ arbitrary cross-linear array, and the array structure used in other algorithms is a $N \times 1$ linear array, where $N=2 M$. We assume that the source signals are nonGaussian and uncorrelated to each other; the Gaussian noises with zero-mean and variance $\sigma^{2}$ are statistically independent of the signals.

An arbitrary cross-linear array is used in the proposed algorithm. To find a suitable cross angle, we compare the performance of all algorithms at different cross angles in following Section 4.1. The influences of different wave directions and different SNR environments on the algorithm are both important factors in the performance evaluation of the DOA estimation algorithm. Thus, the influence of wave direction on the estimation performance is studied in Section 4.2 to detect the estimation accuracy of all algorithms for different wave directions. And simulations under different SNR are implemented.

4.1 Effect of cross-linear angle on estimation performance In the first experiment, all algorithms are compared in terms of RMSE over cross angles. A far-field narrowband signal with an azimuth angle of $30^{\circ}$ incoming with a center frequency of $15 \mathrm{kHz}$. The total sensors number $N$ is 10 , and the SNR is set to $0 \mathrm{~dB}$.

Figure 4a shows that the proposed algorithm perform better as cross angle $\delta$ increases; the performance of other algorithms do not change as $\delta$ increase because only a linear array is used; CRB decreases as $\delta$ increases, which is consistent with the previous analysis of CRB. Besides, the Root-MUSIC algorithm performs rather good when $\Delta c=0$, the MS-KAI-ESPRIT algorithm, and the TLS-ESPRIT algorithm perform moderately, while the AF-ESPRIT algorithm function poorly. And the performance of the proposed VI-LC-ESPRIT algorithm is close to that of AF-ESPRIT when $M=5$ and is close to that of Root-MUSIC when $M=10$.

The AF-ESPRIT algorithm needs to estimate the frequency first, and then use the result of frequency estimation to estimate DOA. Compared with other conventional algorithms, this will bring more errors and lead to worse performance when there is no need to estimate the frequency. Considering that sensors on the $y$-axis in the proposed algorithm is used to eliminate the effect of wave velocity, it does not play a role in reducing the DOA estimation error. Therefore, the proposed algorithm performs worse than other algorithms when $M=5$ and performs better than other algorithms when $M=10$. Also considering that the proposed algorithm performs best when $\delta=90^{\circ}$, the cross angle $\delta$ is set to $90^{\circ}$ in the following experiments.

Figure $4 \mathrm{a}$ and $\mathrm{b}$ illustrate that the proposed algorithm is not affected by the wave velocity. The other algorithms perform worse when $|\Delta c|>0$, and the RMSE of them is larger than $R M S E_{c}$. In addition, CRB at $c=1500 \mathrm{~m} / \mathrm{s}$ is larger than $C R B$ at $c=1475 \mathrm{~m} / \mathrm{s}$, which is consistent with the previous analysis of CRB.

\subsection{Effect of wave direction on estimation performance} In the second experiment, all algorithms are compared in terms of RMSE over wave directions. The $\delta$ is set to $90^{\circ}$

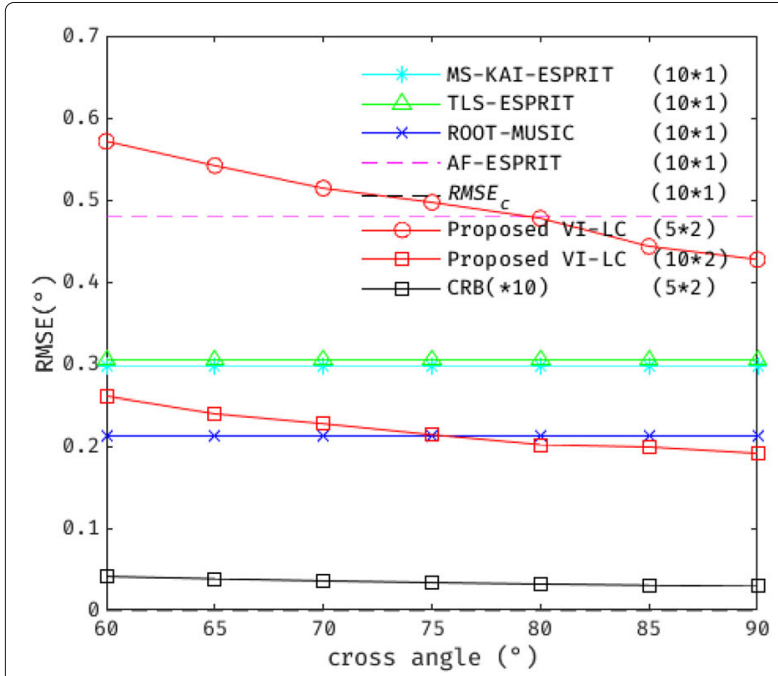

(a)

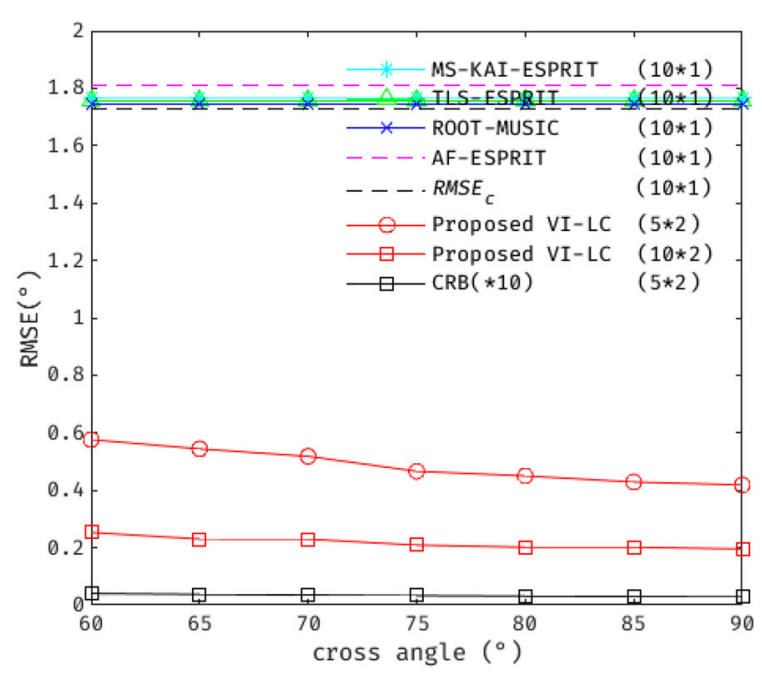

(b)

Fig. 4 RMSE versus cross angles. a $\Delta c=0 \mathrm{~m} / \mathrm{s}$ and $\mathbf{b} \Delta c=-25 \mathrm{~m} / \mathrm{s}$ 
and the other conditions are the same as those of the first experiment.

Figure 5a shows that the performance of the proposed algorithm hardly changes as wave direction increases, while the other algorithms perform better as wave direction $\theta$ increases. According to Eq. (32), $\mathbf{F}_{i i}$ increases as $\theta_{i}$ increases for a single linear array, and $\mathbf{F}_{i i}$ does not change significantly for the arbitrary cross-linear array. Because of $\mathbf{C}=\mathbf{F}^{-1}$, it means that the performance of the proposed algorithm is not affected significantly as $\theta$ increases, and the other algorithms perform better as $\theta$ increases, which is consistent with the experimental results.

From Fig. $5 \mathrm{a}$ and $\mathrm{b}$, it can be noted that the estimation performance of the proposed algorithm is well in different wave velocity environments than other algorithms. Besides, the other algorithms perform worse when $|\Delta c|>$ 0 than that when $|\Delta c|=0$. The simulation result shows the proposed algorithm maintains robust performance in an unknown wave velocity environment.

\subsection{Comparison of algorithms with different sNR}

In the third experiment, the proposed VI-LC-ESPRIT algorithm in theoretical analysis and experimental studies, MS-KAI-ESPRIT algorithm, TLS-ESPRIT algorithm, Root-MUSIC algorithm, AF-ESPRIT algorithm, and CRB are compared in term of RMSE with respect to SNRs in an Gaussian noise situation. The number of sensors $N$ is set to 10 . Two uncorrelated equal power signals with azimuth angles $\theta$ and frequency from $\left(30^{\circ}, 60^{\circ}\right)$ and $(15000,16000) \mathrm{kHz}$.

From Fig. 6a, it can be noted that the RMSE of all algorithms, except MS-KAI-ESPRIT, and the CRB decreases as SNR increases. The proposed algorithm performs better than the others at high SNR, while it is worse at low SNR. Considering that sensors on the $y$-axis in the proposed algorithm is used to eliminate the effect of wave velocity, it does not play a role in reducing the DOA estimation error. The proposed algorithm performs better than other algorithms at high SNR when using $10 \times 2$ sensors. Besides, because the TLS-ESPRIT algorithm and the Root-MUSIC algorithm are not suitable for DOA estimation of multi-frequency sources, their performance is worse than the AF-ESPRIT algorithm when $\Delta c=0$.

Figure $6 \mathrm{a}$ and $\mathrm{b}$ note that the estimation performance of the proposed algorithm at $\Delta c=0$ is roughly the same as that at $\Delta c=-25 \mathrm{~m} / \mathrm{s}$, while other algorithms perform worse as $\Delta c$ increases. Also, the performance of AF-ESPRIT is close to $R M S E_{c}$ at high SNR, which quite matches the theoretical analysis in Eq. (7).

\subsection{Comparison of algorithms with unknown wave velocity}

In the fourth experiment, the above algorithms are compared in terms of RMSE with respect to the real-time wave velocity. The parameters configured in this experiment are the same as the third experiment.

From the result of Fig. 7a, the proposed algorithm maintains robust performance as $\Delta c$ increases, and far superior to the other algorithms when $M=10$. The larger $|\Delta c|$ is, the worse the estimation performs among other algorithms. And the performance of the AF-ESPRIT is close to $R M S E_{c}$ which is better than the classical algorithms. Besides, the MS-KAI-ESPRIT algorithm performs worse at multi-frequency and inaccurate velocity environments.

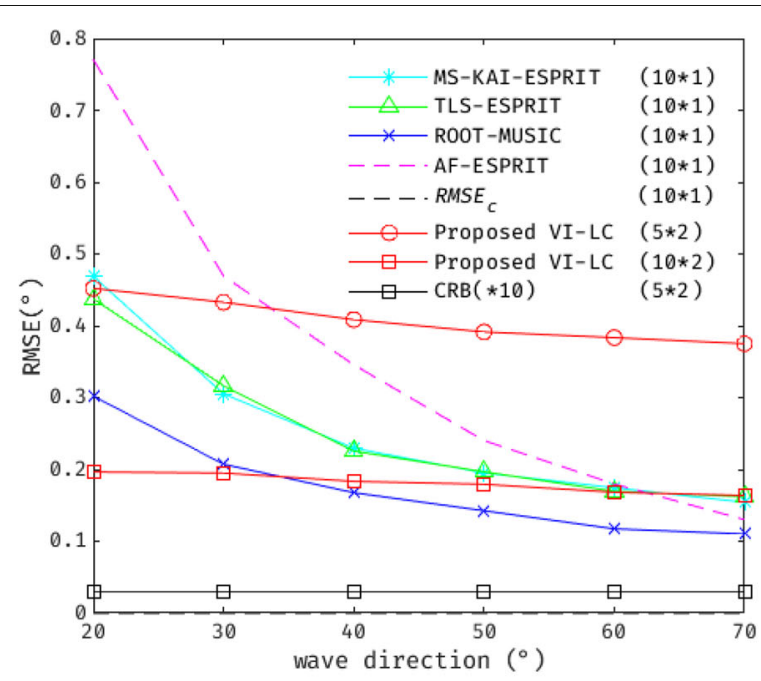

(a)

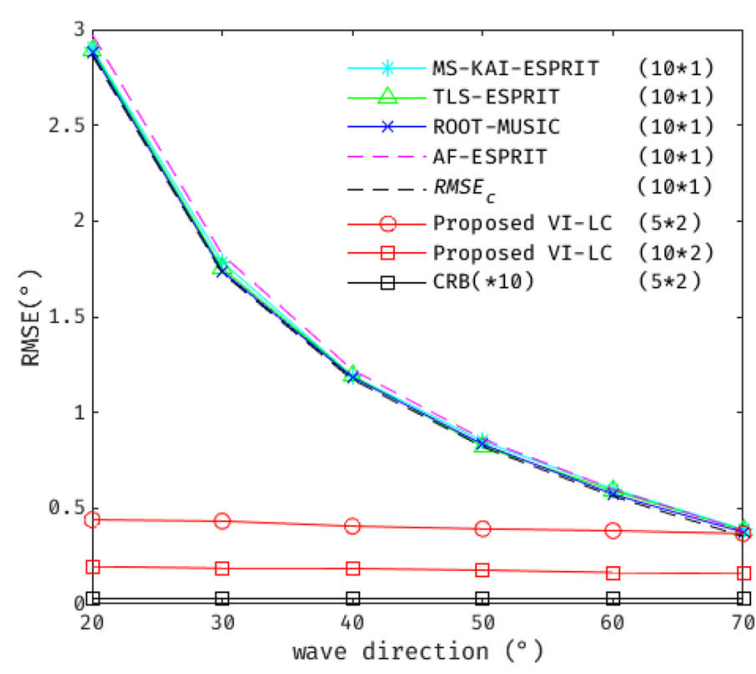

(b)

Fig. 5 RMSE versus wave directions. a $\Delta c=0 \mathrm{~m} / \mathrm{s}$ and $\mathbf{b} \Delta c=-25 \mathrm{~m} / \mathrm{s}$ 


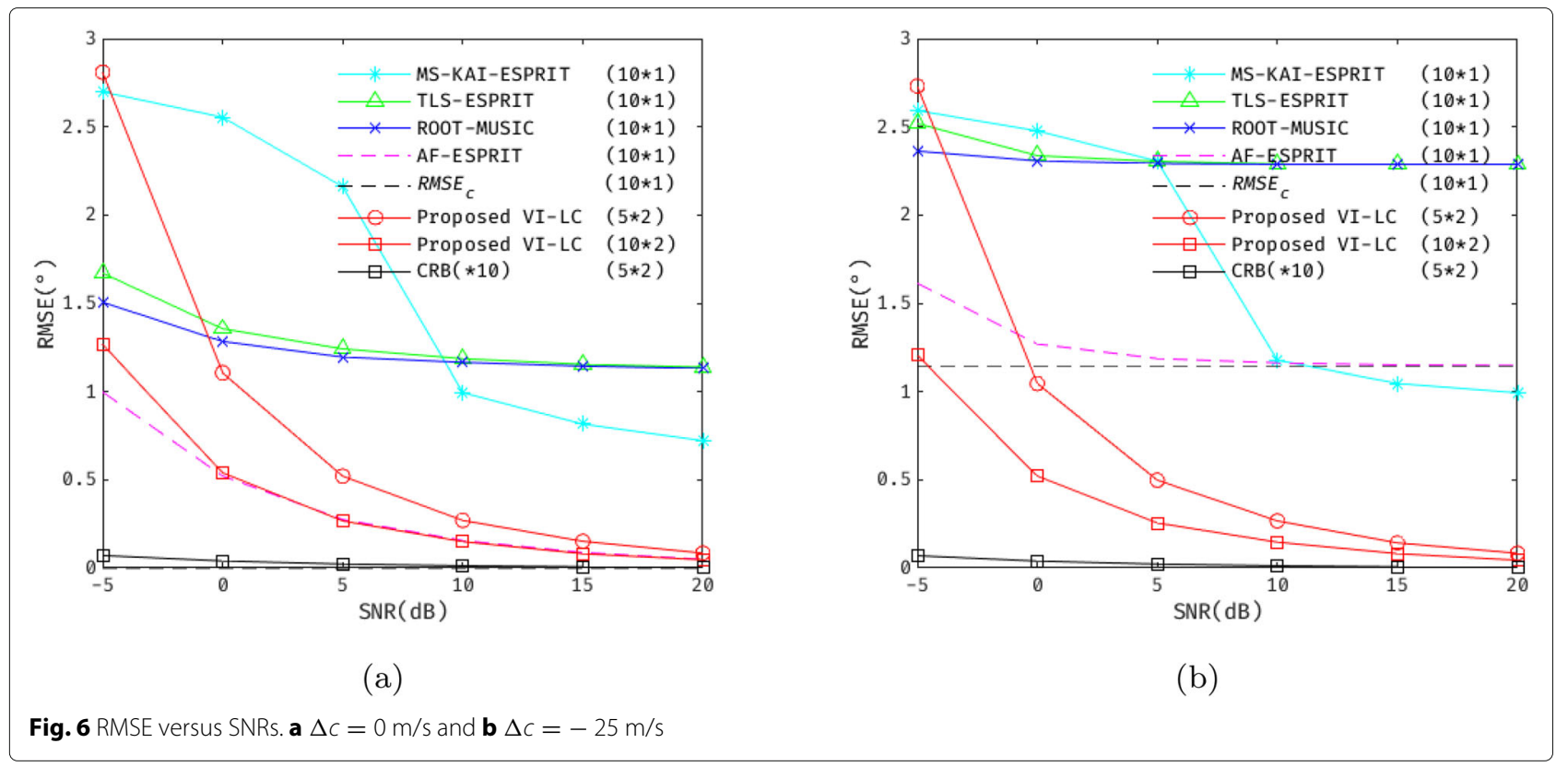

Figure $7 \mathrm{a}$ and $\mathrm{b}$ note that the estimate performance of the proposed algorithm is better at $\mathrm{SNR}=10 \mathrm{~dB}$ than that at $\mathrm{SNR}=0 \mathrm{~dB}$ and the proposed algorithm is also better than others when $|\Delta c|>0$.

\section{Conclusions}

This paper proposes a velocity-independent and lowcomplexity 1D-DOA estimation algorithm with an arbitrary cross-linear array to solve the problem of unknown underwater wave velocity. The arbitrary cross-linear array is employed to reduce the estimation error caused by assuming a fixed wave velocity. Additionally, the proposed algorithm has low complexity, for it does not require parameter pairing. Compared with the traditional 1D ESPRIT algorithm (e.g., AF-ESPRIT and MS-KAIESPRIT), it can deal with the effect of wave velocity deviation and the computational complexity is much lower than that of the former VI-MUSIC algorithm. The simulation results well coincide with the analysis of the $R M S E_{c}$ and the CRB. Simulation experiments bear out the proposed VI-LC-ESPRIT algorithm, which is effective in handling the impact of an inaccurate wave velocity value.

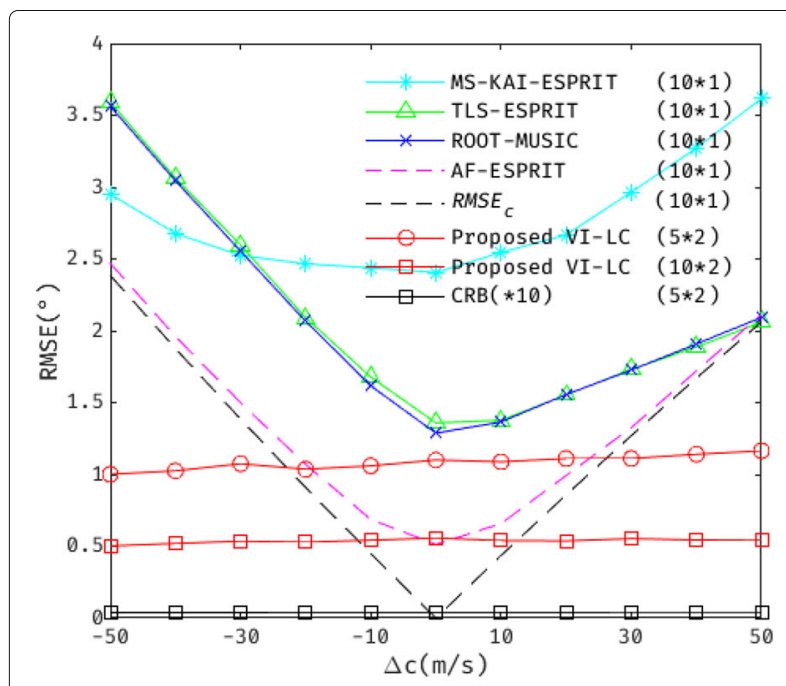

(a)

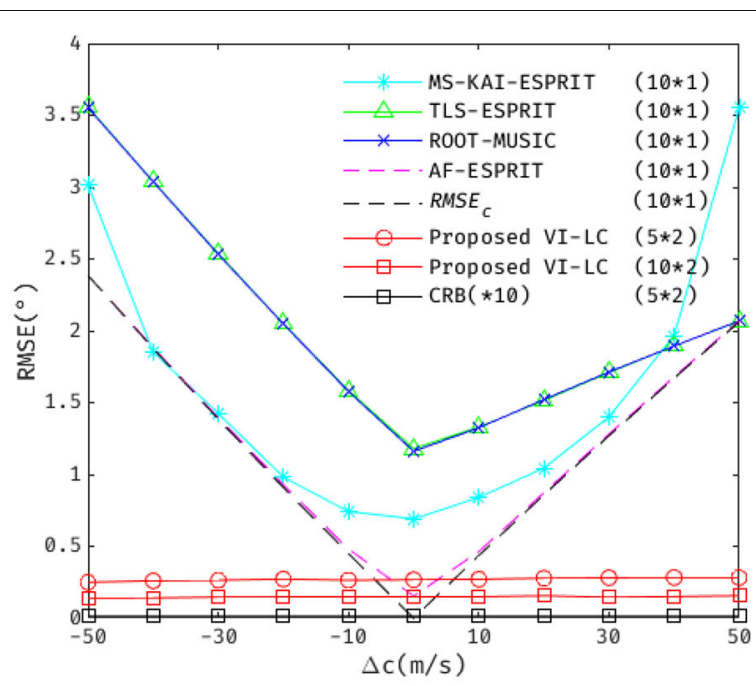

(b)

Fig. 7 RMSE versus $\Delta c$. a SNR $=0 \mathrm{~dB}$ and $\mathbf{b} S N R=10 \mathrm{~dB}$ 


\section{Abbreviations}

MR-ESPRIT: Manifold reconstruction estimating signal parameter via rotational invariance techniques; AF-ESPRIT: Joint angle and frequency estimating signal parameter via rotational invariance techniques; MS-KAI-ESPRIT: Multistep knowledge-aided iterative estimating signal parameter via rotational invariance techniques; TLS-ESPRIT: Total least square estimating signal parameter via rotational invariance techniques; VI-MUSIC: Velocity-independent multiple signal classification; VI-LC-ESPRIT: Velocity-independent and low-complexity estimating signal parameter via rotational invariance techniques algorithm proposed in this paper;

\section{Acknowledgements}

The authors would like to thank all anonymous reviewers and editors for their helpful suggestions for the improvement of this paper.

\section{Authors' contributions}

GN and XL proposed the original idea of the full text; GN and GJ designed and implemented the simulation experiments; GJ and XZ wrote the manuscript under the guidance of GN. All authors read and approved this submission.

\section{Funding}

This work supported by the National Natural Science Foundation of China (61871191), Science and Technology Planning Project of Guangdong Province (2016A020222003,2017A030313368), and Science and Technology Planning Project of Guangzhou (201804010209).

\section{Competing interests}

The authors declare that they have no competing interests.

\section{Author details}

${ }^{1}$ South China University of Technology, Guangzhou, China. ${ }^{2}$ Guangdong University of Technology, Guangzhou, China.

Received: 12 January 2020 Accepted: 2 June 2020

Published online: 16 June 2020

\section{References}

1. D. Sun, C. Zheng, J. Zhang, Y. Han, H. Cui, Development and prospect for underwater acoustic positioning and navigation technology. J. Chin. Acad. Sci. 34(3), 331-338 (2019)

2. S. M. Yusop, M. N. A. Bakar, K. Abdullah, in 2011 IEEE Colloquium on Humanities, Science and Engineering. Kuala Besar, Kelantan: a sound speed analysis (IEEE, 2011). https://doi.org/10.1109/chuser.2011.6163815

3. R. Roy, T. Kailath, ESPRIT-estimation of signal parameters via rotational invariance techniques. IEEE Transactions on acoustics, speech, and signal processing. 37(7), 984-995 (1989)

4. C. Qian, A simple modification of ESPRIT. IEEE Signal Processing Letters. 25(8), 1256-1260 (2018)

5. X. Wang, X. Zhang, J. Li, J. Bai, Improved ESPRIT method for joint directionof-arrival and frequency estimation using multiple-delay output. Int. J. Antennas Propag. 2012, 1-9 (2012). https://doi.org/10.1155/2012/309269

6. L. Liu, P. Wei, Joint DOA and frequency estimation with sub-Nyquist sampling in the sparse array system. IEEE Sig. Process. Lett. 25(9), 1285-1289 (2018)

7. S. F. Pinto, R. C. De Lamare, Multistep knowledge-aided iterative ESPRIT: design and analysis. IEEE Trans. Aerosp. Electron. Syst. 54(5), 2189-2201 (2018)

8. J. Liang, D. Liu, Joint elevation and azimuth direction finding using L-shaped array. IEEE Trans. Antennas Propag. 58(6), 2136-2141 (2010)

9. N. Xi, L. Li, A computationally efficient subspace algorithm for 2-D DOA estimation with L-shaped array. IEEE Sig. Process. Lett. 21(8), 971-974 (2014)

10. J. Ding, M. Yang, B. Chen, X. Yuan, A single triangular SS-EMVS aided high-accuracy DOA estimation using a multi-scale L-shaped sparse array. EURASIP J. Adv. Sig. Process. 2019(1), 44 (2019)

11. X. Zhang, C. Chen, J. Li, D. Xu, Blind DOA and polarization estimation for polarization-sensitive array using dimension reduction MUSIC. Multidim. Syst. Signal Process. 25(1), 67-82 (2014)

12. A. Liu, F. Li, B. Li, Q. Liu, X. Shi, Spatial polarimetric time-frequency distribution based DOA estimation: combining ESPRIT with MUSIC. EURASIP J. Wirel. Commun. Netw. 2018(1), 1-8 (2018)
13. Z. Zhang, Y. Guo, Y. Huang, P. Zhang, A 2-D DOA estimation method with reduced complexity in unfolded coprime L-shaped array. IEEE Syst. J., 1-4 (2019). https://doi.org/10.1109/jsyst.2019.2948089

14. X. Zhang, W. Zhang, Y. Yuan, K. Cui, T. Xie, N. Yuan, DOA estimation of spectrally overlapped LFM signals based on STFT and Hough transform. EURASIP J. Adv. Sig. Process. 2019(1), 1-10 (2019)

15. G. Ning, B. Wang, C. Zhou, Y. Feng, in 2017 IEEE International Conference on Signal Processing, Communications and Computing (ICSPCC). A velocity independent MUSIC algorithm for DOA estimation (IEEE, 2017). https:// doi.org/10.1109/icspcc.2017.8242414

16. Petre Stoica and Randolph Moses, Spectral analysis of signals. (Pearson Prentice Hall Upper Saddle River, NJ, 2005), pp. 355-367

17. X. Yang, T. Long, T. K. Sarkar, in IEEE 1Oth International Conference On Signal Processing Proceedings. Effect of geometry of planar antenna arrays on Cramer-Rao bounds for DOA estimation (IEEE, 2010). https://doi.org/10. $1109 /$ icosp.2010.5657183

\section{Publisher's Note}

Springer Nature remains neutral with regard to jurisdictional claims in published maps and institutional affiliations.

\section{Submit your manuscript to a SpringerOpen ${ }^{\circ}$ journal and benefit from:}

- Convenient online submission

- Rigorous peer review

- Open access: articles freely available online

- High visibility within the field

- Retaining the copyright to your article

Submit your next manuscript at $>$ springeropen.com 